\title{
Osteoporosis in High Income Kashmiri Population - A Tertiary Care Centre Study
}

Authors

\author{
Mohd Ahsan Wani ${ }^{1}$, Mushtaq Ahmed Ganaiee ${ }^{2}$, Akther Rasool ${ }^{3}$, Muneeb UI Islam ${ }^{4}$ \\ ${ }^{1,2}$ Senior Resident Orthopaedics GMC Srinagar, India \\ ${ }^{3,4}$ Post Graduate Student GMC Srinagar, India
}

\begin{abstract}
Various factors, including unbalanced diet and hormonal disturbances have been attributed to the presence of osteoporosis in individuals of elite society. A study of clinical profile of osteoporosis in elite population in Kashmiri population was carried out at the Bone and Joint Hospital Barzullah Srinagar. 100 patients were selected for the study on the basis of DEXA bone density findings.
\end{abstract}

\section{Introduction}

People with access to good nutrition, a decent environment and good medical facilities and have decent knowledge about prevention \& treatment of various diseases are included in well of population group so called ellite group. 'Female Athlete Triad', a term coined in 1992 by the American College of Sports ${ }^{1,2,3.4}$ Medicine, in response to several studies found high incidence of this entity in elite female athletes. The observations from International Olympic Committee Medical forum established that osteoporosis $^{5,6}$ can be found even in otherwise well-to-do people with sound knowledge of diet and ample resources. A study of osteoporosis in the higher socio-economic group was carried out in patients reporting at the Bone and Joint Hospital Barzullah Srinagar.

\section{Material and Methods}

100 cases of Osteoporosis attending the Out Patient's and in-patient's Department of the
Orthopaedics GMC Srinagar, were selected for the study. The inclusion criteria were (1) cases of osteoporosis with varying etiology, (2) patients belonging to higher income group. A detailed clinical history was taken with special attention history of taking drugs - thyroid medication, steroid based drugs for asthma, arthritis or cancer, anti-epileptics, chronic heparin administration, and the history of disorders in which osteoporosis is a common feature, such as diabetes mellitus and hypothyroidism; to alcohol intake, smoking, sedentary life style, chronic intestinal disorders, in females also menstrual history, including the history of late menarche or early menopause, surgery on uterus and ovaries was also taken. The gold standard for the diagnosis was DEXA bone densitometry of Lumbar spine and proximal femur to confirm and quantify osteoporosis. X-ray of lumbosacral spine and pelvis with both hip joints was also taken in each case. ${ }^{7,8}$ The other investigations included hemoglobin ( $\mathrm{Hb})$, erythrocyte sedimentation rate (ESR), blood 
sugar, serum calcium, serum Osteoporosis in Elite Population, phosphorous, alkaline phosphatase, 24-hour urinary excretion of calcium. Special investigations, wherever indicated, were done, such as serum electrophoresis for myeloma proteins, thyroid stimulating hormone.

\section{Observations}

Age and Sex: There were 25 males and 75 females in this study. The age of patients ranged from 35 to 85 years. Maximum number of patients was present in the age groups 51-60 years and 6170 years. number of females presenting with osteoporosis is larger than males, which is statistically significant.

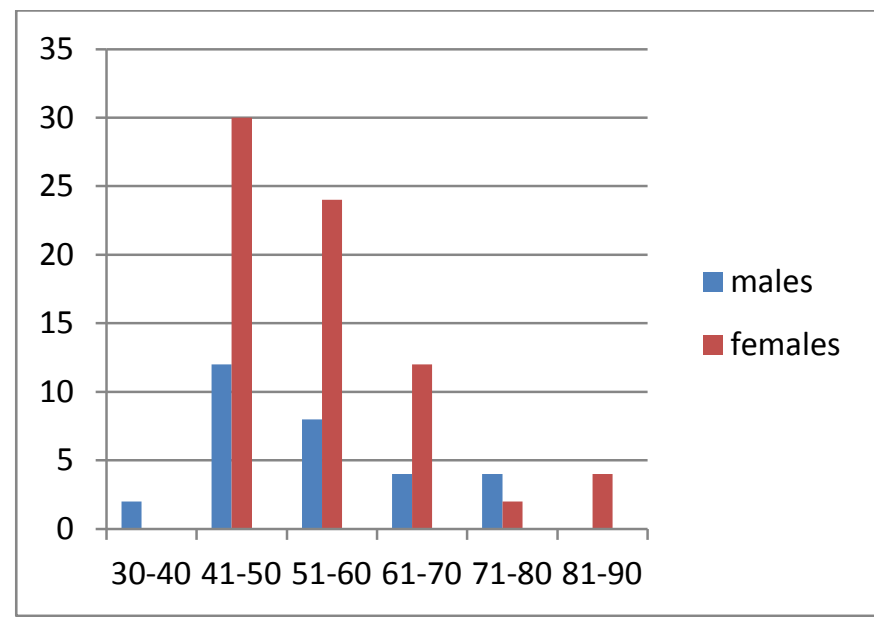

Histogram-1: Distribution of males and females with osteoporosis in the Histogram

Fracture risk: The relation between the risks of fracture as assessed from the DEXA report is shown Table 1. The fracture risk was reported as mild (risk 1), moderate (risk 2) and severe (risk 3).

\begin{tabular}{|l|c|c|c|}
\hline Fracture risk & 1 mild & 2moderate & 3severe \\
\hline cases & 6 & 44 & 50 \\
\hline Fracture present & 2 & 19 & 4 \\
\hline
\end{tabular}

Twenty five patients out of the total of 100 cases had presented with fracture. A correlation was attempted between the fracture risks as per the DEXA findings. 15 patients had compression fracture of vertebra, 5 patients had fracture neck of femur, 3 patients had Colles' fracture and 2 had fracture of the shaft of humerus. The patient presenting with the fracture of humerus, Colles' fracture and fracture neck of femur had a history of fall or had typical deformity but no patient of spinal fracture gave any history of significant injury. The patients with the fracture of humerus, Colles fracture, and fracture of neck of femur had typical deformity. On the other hand the patients with spine fracture mainly presented with localized backache. No patients had signs of neurological deficit. Backache: The usual presentation of the patients was low backache, but some patients complained of generalized body ache. Low backache was the main presenting feature of milder cases of osteoporosis.

Generalized body ache was a prominent presentation in moderate and severe osteoporosis. While pain responded to analgesics in milder cases, many advanced cases who did not respond to the usual analgesics, nasal insufflations of Calcitonin spray was very helpful in some cases. High calcium intake, gradual exercise, and a balanced diet greatly helped in ameliorating the symptoms, though no significant improvement in $\mathrm{X}$-ray picture or DEXA findings was noted during the period of study.

Neurological involvement: No patient had overt neurological deficit in this study in spite of collapse of vertebrae. Patients with collapse of vertebrae had pain in legs and paraesthesias. Patient's with collapse of vertebra were advised extension braces to be worn when the patient was ambulatory to prevent further collapse of the vertebrae and hence preventing improvement of neurological symptoms.

Bone density (DEXA) and Singh Index: Plain radiographs of trabecular bone show a distinct pattern of differences in the appearance of healthy and osteoporotic bone. We therefore evaluated Singh index 26 against the findings of DEXA of proximal femur. The Singh index has been evaluated against 1 standard deviation (1 SD), 12.5 standard deviation (1-2.5 SD) and less than/equal to 2.5 standard deviation (2.5 SD and below) of DEXA findings. However, the DEXA findings of lumbaspine ( $\mathrm{L} 1$ to $\mathrm{L} 4$ ) revealed established osteoporosis (DEXA T-values of 
lumbar spine had SD <2.5). We therefore conclude that the Singh Index has poor reliability and poor diagnostic value in screening patients of osteoporosis, especially with respect to spine Causes of osteoporosis and coexistent diseases: The cause of osteoporosis in the present study comprised of multiple myeloma, post menopausal, idiopathic, unbalanced diet and lack of physical activity. Co-existent diseases in this group, which included diabetes mellitus, hypertension. coronary artery disease (CAD), hypothyroidism; detailed evaluation of nutritional status was not feasible in the present study. However, the dietetic history revealed high incidence of unbalanced diet, which was rich in calories but deficient in calcium and protein.

Table 2 shows the chronic medical conditions associated with osteoporosis in this study.

\begin{tabular}{|l|c|}
\hline Associated diseases & Number of patients \\
\hline Bronchial asthama & 4 \\
\hline CAD & 4 \\
\hline DM & 20 \\
\hline HT & 20 \\
\hline Hypothyroidism & 6 \\
\hline Multiple myeloma & 2 \\
\hline Valvular Heart diseases & 1 \\
\hline Total & 40 \\
\hline
\end{tabular}

Out of a total of 100 patients of osteoporosis, 40 had one or more associated diseases. These diseases included bronchial asthma (4case), coronary artery disease (4 case), diabetes mellitus (20cases) hypertension (20 cases), hypothyroidism (6 cases), multiple myeloma ( 2 case) and valvular heart disease ( 1 case). Of these 40 patients 12 had two or more associated diseases. For this reason, the number of associated diseases is more than the total number of patients with associated diseases.24-Hour Urinary Calcium: Urinary calcium in patients in this series is given in the Histogram 3. Normal excretion of urinary calcium was taken as less than $150 \mathrm{mg}$ in 24 hours at normal dietary intake of calcium [Harrison's Principles of Internal Medicine].94 patients (94\%) had urinary excretion of calcium less than $150 \mathrm{mg}$ in 24 hours. But 6 patients $(6 \%)$ were found to excrete calcium more than 150 cut-off limit. 4 of these patients had urinary calcium of 158 and 160 $\mathrm{mg} / 24 \mathrm{hrs}$, which are not significantly elevated from the normal value of $150 \mathrm{mg} / 24 \mathrm{hrs}$. Only one patient had urinary calcium $211.6 \mathrm{mg} \%$. This patient had diabetes mellitus as an associated disease. There was no hypercalcemia in this patient (serum calcium being $9.2 \mathrm{mg} \%$ ).

Histogram 3: Urine Calcium excretion

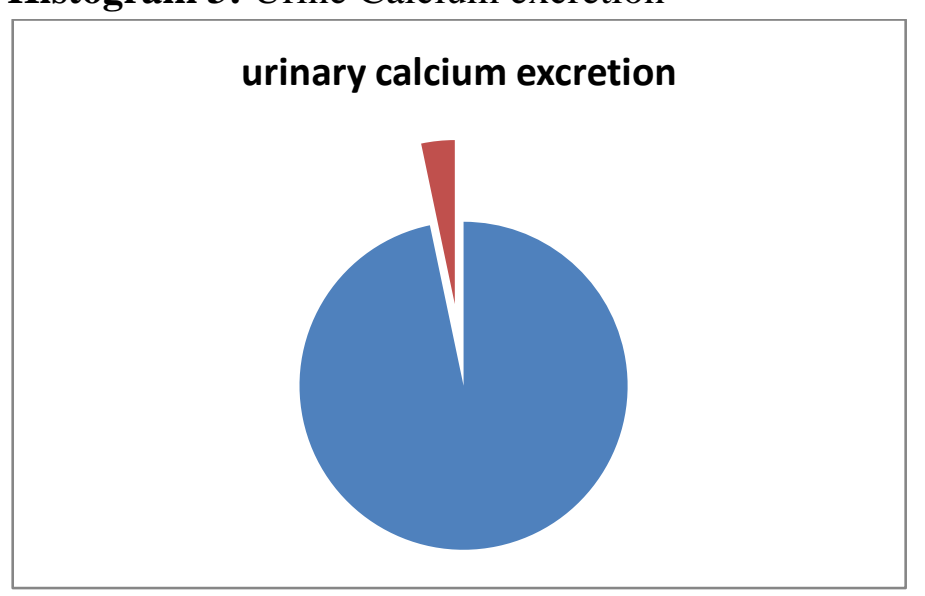

\section{Discussion}

Incidence of Osteoporosis

The findings of age and sex are given in Histogram-1. It will be noted that the number of females presenting with osteoporosis is larger than males. The amount of calcium loss in males may be lower on account of their more vigorous physical activity. Studies have shown that accumulation of calcium in bone is greatly influenced by physical activity ${ }^{10}$.

In general, the females were housewives in this higher income group. They had lot of domestic help to look after their routine work, which further curtailed their physical activity. Further, the hormonal disturbances in the perimenopausal period are known to influence bone mineral concentration $^{11,12}$. The peak bone-mass attained during adolescence is an important determinant of bone density in the later period of life ${ }^{13}$ The boys being more physically active tend to attain higher peak-bone mass during the adolescent period, thereby influencing the development of osteoporosis in the later age group. Causes of osteoporosis In our study the maximum number of patients had diabetes mellitus. The other two conditions forming the bulk of association in our 
series, namely the CAD and hypertension, have no direct relation with osteoporosis.

Weinstock et al (1989) ${ }^{14}$ found no correlation between BMD and diabetes duration or current glycemic control in postmenopausal women ${ }^{15}$. Short-term measures of control, such as glucose levels or HBA1C results, have also not been found to reflect cumulative bone damage measured by BMD Valerio et $a^{116}$. The complications of diabetes may represent cumulative results of longterm poor control. Several studies have demonstrated an association between BMD and microvascular complications ${ }^{14}$. In a prospective study reported that after 11 years, only those subjects who developed retinopathy or proteinuria had worsening of their BMD ${ }^{17}$. Forst et al. ${ }^{18}$ found a decreased BMD in the cortical bone at the hip and a greater decrease at the distal limb in association with peripheral neuropathy. The lumbar spine had a non significant decrease in BMD in these patients with Type- 1 diabetes $1^{9,20}$ The Blue Mountain Eye Study in Australia ${ }^{21}$ found an association between retinopathy and all fractures in both men and women with all types of diabetes ${ }^{19,20}$. Some studies have shown stabilized and improved BMD in patients with type 1 diabetes with improved glucose control over time $1^{9,}$ 22. Hypercalciuria, a potential risk factor for osteoporosis, has long been noted in patients with poorly controlled Type- 1 or Type- 2 diabetes, and was shown to improve with lower A1C results $^{21,23}$. None of our patient had Type-I diabetes mellitus. Although it is well known that diabetes mellitus favours the development of osteoporosis, none of our patients had longstanding diabetes which can be considered as a causative factor. The incidence of coronary artery disease, hypertension and diabetes mellitus, is known to increase with advancing age. Their presence in large number in the present series cannot be related to as causative factors. Prolonged use of steroid therapy is also well known to cause osteoporotic changes, but none of our patients had sufficiently long intake of oral steroids to account for causation of osteoporosis.
Multiple myeloma was detected in two patients. However, the lesions were localized to spine which presented as vertebral compression fractures and generalized osteoporosis. We therefore conclude that association of diseases in the present series was casual and did not seem to have any direct cause-effect relationship. It is however possible that these diseases could have contributed to ongoing osteoporotic process. Urinary Calcium: In this regard it is worth mentioning a study of urinary calcium in osteoporosis carried out by Bhandarkar and Nordin $^{24}$. They found a significant difference between the ability of normal subjects and patients with osteoporosis to reduce their urinary calcium excretion. In the normal subjects the mean excretion of calcium fell significantly on low calcium diet, but in the osteoporotic cases it did not. They could not conclude with confidence whether the failure of osteoporotic subject to reduce urinary calcium excretion was a cause or the effect of osteoporosis, they concluded on the basis of their previous observations that the former was inherently more possible. They concluded from their studies 25 that there is no relation between dietary calcium and urinary calcium in osteoporosis. But osteoporosis may be due to disturbances in renal handling of calcium, the cause of which could be obscure, but it might be connected with the alleged role of oestrogenic hormones or might perhaps be connection with high incidence of infection of urinary tract infection in women. We could not work out the calcium balance studies as the patient left the hospital. But it is proposed that a careful observation of renal handling of calcium deserve to be studied in greater detail in all patients of osteoporosis. Singh Index To underline the usefulness of this pattern in osteoporosis, the Singh index grading system was devised in the 1960s by using radiographs of the proximal femur $^{26}$. It is based on the radiological appearance of the trabecular bone structure of the proximal femur on a plain anteroposterior radiograph. The Singh index is used to assess patterns of trabecular 
loss. Dual-energy X-ray absorptiometry (DEXA) provides a more precise estimate of bone mineral density $^{27,28,29}$ and is considered to be the 'gold standard' for measuring osteoporosis ${ }^{30}$. Koot et al. ${ }^{31}$ assessed the inter-observer and intra-observer agreement of readings of the Singh index and compared the indices for measuring osteoporosis ${ }^{30}$ with the results of DEXA. They did not find any good relation between DEXA and Singh Index ${ }^{31}$. As per the original description by Singh et al, the grade-1 represents the severest form of osteoporosis. .

Singh Index has been recently criticized for its low reliability due to the subjective nature of its ill defined grading and cut-off level for osteoporosis $^{27,28,29,30}$. Currently DEXA is considered as the gold-standard for diagnosis of osteoporosis. The commonly acknowledged medical risk factors for osteoporosis are chronic liver disease, chronic renal failure, thyroid disease, particularly hyperthyroidism or excessive thyroxin replacement, primary hyperparathyroidism, prolonged immobilization, Cushing's syndrome or disease, Corticosteroid therapy, Malabsorptive illnesses

\section{References}

1. Otis CL, Drinkwater B, Johnson M: American College of Sports Medicine position stand: The Female Athlete Triad. Med Sci Sports Exerc 1997, 29(5): i-ix.

2. Wilmore JH: Relation of level of exercise, age, and weight-cycling history to weight and eating concerns in male and female runners. Health Psychology 1992, 11:418421.

3. Yeager KK, Agostini R, Nattiv A, Drinkwater B: The female athlete triad: disordered eating, amen-orrhea, osteoporosis. Med Sci Sports Exerc 1993, 25(7):775-777.

4. OC Medical Commission Working Group Women in Sport (IOCMC): Position Stand on the fe-male athlete triad [http:/ / www.olympic.org/ common/ asp/ download_report.asp?file=en_report_917.pd f\&id=917] 2005.

5. Thangaleela, T; Vijayalakshmi, P (1994): Prevalence of Anaemia in Pregnancy. The Indian Jour-nal of Nutrition and Dietetics. Feb 1994. 31(2). p.26-29.

6. Miller RG, Segal JB, Ashar BH, Leung S, Ahmed S, Siddique $S$, et al . High prevalence and correlates of low bone mineral density in young adults with sickle cell disease. Am J Hematol 2006; 81:23641.

7. Faber TD, Yoon DC, White SC. Fourier analysis reveals increased trabecular spacing in sickle cell anemia. J Dent Res 2002; 81:214-8.

8. Gurevitch O, Slavin S. The hematological etiology of osteoporosis. Med Hypotheses. 2006; 67:729-35.

9. Groopman JE., Itri LM. ChemotherapyInduced Anemia in Adults: Incidence and Treatment. Jour-nal of the National Cancer Institute, Vol. 91, No. 19, 1616-1634, October 6, 1999.

10. J Zhang, P J Feldblum, and J A Fortney. Moderate physical activity and bone density among perimenopausal women. Am J Public Health. 1992 May; 82(5): 736-738.

11. Zumoff B, Strain GW, Miller LK et al. Twenty-four hour mean plasma testosterone concentration declines with age in normal premenopausal women. J Clin Endocrinol Metab. 1995; 80:1429-1430.

12. Chapurlat RD, Gamero P, Sornay-Rendu E et al. Longitudinal study of bone loss in preand perimenopausal women: evidence for bone loss in perimenopausal women. Osteoporos Int. 2000; 11:493-498.

13. Seeman E. Pathogenesis of bone fragility in women and men. The Lancet. 2002; 359; 1841-1850.

14. Weinstock RS, Goland RA, Shane E, Clemens TL, Lindsay R. Bilezikin JP: Bone mineral density in women with type II 
diabetes mellitus. J Bone Miner Res. 4: 97101, 1089].

15. Riggs BL, Hodgson SF, O'Fallon WM, Chao EY, Wahner HW, Muhs JM, et al. Effect of fluoride treatment on the fracture rate in postmenopausal women with osteoporosis N Engl J Med. 1990; 322:8029].

16. Pak CY, Shakhaee K, Piziak V, Peterson RD, Breslau NA, Boyd P, et al. Slow release sodium fluoride in the management of postmenopausal osteoporosis. A randomized controlled trial. Ann Intern Med. 1994; 120:624-232]).

17. Valerio G, del Puente A, Esposito-del Punte A, Buono P, Mozzillo E, Franzese A: The lumbar bone mineral density is affected by long-term poor metabolic control in adolescents with type I diabetes mellitus. Horm Res. 58:266-272, 2002].

18. Forst T, Pfutzner A, Kann P, Schehler B, Lobmann R, Schafer H, Andreas J, Bockisch A, Beyer J: Peripheral osteopenia in adult patients with insulin-dependent diabetes mellitus. Diabet Med. 12:874-879, 1995]

19. Tuominen JT, Impivaara O, Puukka P, Ronnemaa $\mathrm{T}$ : Bone mineral density in patients with type 1 and type 2 diabetes. Diabetes Care. 22:1196-1200, 1999]

20. Christensen JO, Svendsen OL: Bone mineral in pre- and postmenopausal women with insulin-dependent and non-insulin dependent diabetes mellitus. Osteoporosis Int. 10:307-311, 1999.

21. Ivers RQ, Cumming RG, Mitcechell $P$, Peduto AJ: Diabetes and risk of fracture: the Blue Moun-tain Eye Study. Diabetes Care. 24: 1198-1203, 2001].

22. Mathiassen B, Nielsen S, Ditzel J, Rodbro P: Long-term bone loss in insulin dependent diabetes mellitus. J Intern Med. 227:325327,1990

23. Krakauer JC, McKenna MJ, Buderer NF, Rao DS, Whitehouse FW, Parfitt AM: Bone loss and bone turnover in diabetes. Diabetes 44:775-782m 1995]

24. Bhandarkar S.D and Nordin B.E.C (1962): Effect of low calcium diet on urinary calcium in osteoporosis. Brit. Med. J.; I (5272):145-147

25. Nordin, B.E.C: Lancet. 1961; 1:1011 (Quoted by Bhandarkar and Nordin (1962).

26. Singh M, Nagrath AR, Maini PS. Changes in trabecular pattern of the upper end of the femur as an index of osteoporosis. J. Bone Joint Surg [Am]. 1970; 52-A:457-67

27. Kawashima T, Uhthoff HK. Pattern of bone loss of the proximal femur: a radiologic, densitometric and histomorphometric study. J Orthop Res. 1991; 9:634-40.

28. 28. Lang P, Steiger P, Faulkner K, Gluer C, Genant HK. Osteoporosis: current techniques and recent developments in quantitative bone densitometry. Radiol Clin North Am. 1991; 29:49-76.

29. Prince RL, Geelhoed E, Harris T, et al. Screening for osteopososis by bone densitometry: a striatified intervention approach. Osteoporosis Int. 1993; 3: Suppl 1:75-77.

30. Sartoris DJ. Clinicla value of bone densitometry. Am J Roengenol, 1991; 163:133-35.

31. Koot V.C.M., Kesselaer S.M.M.J., Clevers G. J., Hooge P. De, Weits T., Werken Chr. Van Der. Evaluation of the Singh index for Measuring osteoporosis. J Bone Joint Surg [Br]. 1996; 78-B:831-834.

32. Kinoshita T, Ebara S, Kamimura M, et al. Nontraumatic lumbar vertebral compression fracture as a risk factor for femoral neck fractures in involutional osteoporotic patients. J Bone Miner Metab.1999; 17(3):201-5

33. Seeman E. Reduced bone formation and increased bone resorption: rational targets for the treat-ment of osteoporosis. Osteoporos Int. 2003; 14 Suuppl 3:S2-8. 